\title{
To Study the Safety of Multi-dose Topical Anaesthetic Eye Drops For One Month after Opening
}

\author{
Mehr-un-Nisa, Irfan Qayyum Malik, Muhammad Wasif Irshad
}

Pak J Ophthalmol 2019, Vol. 35, No. 3

See end of article for

authors affiliations

Correspondence to:

Mehr-un-Nisa

Department of Ophthalmology

DHQ Teaching Hospital,

Gujranwala

Email: 06mehru@gmail.com
Purpose: To determine the risk of ocular infections due to topical multi-dose anaesthetic eye drops in patients one month after opening the bottle.

Study Design: Quasi experimental study.

Place and duration of study: Department of Ophthalmology DHQ Teaching Hospital, Gujranwala from $22^{\text {nd }}$ May 2018 to $22^{\text {nd }}$ June 2018.

Materials and Methods: In this study topical anaesthetic eye drops containing proparacaine hydrochloride $0.5 \%$ as main ingredient and benzalkonium choride as preservative (Alcaine ${ }^{\circledR}$ ) were tested daily to see if any bacterial/fungal growth occurs in the bottle containing eye drops or not. Same bottle was used in patients presenting in outdoor and indoor departments. Samples taken from the bottle contents were cultured on different media to see growth of fungi or bacteria. Patients who already had keratitis, conjunctivitis, corneal opacities, thin corneas and having dry eyes which were prone to get infected were excluded from this study.

Results: There were 60 patients included in our study and they were divided in two groups. Group A included those who presented in the outdoor department and group B comprised of those admitted for various operative procedures. There were 30 patients in each group. All patients were adults (18-60 years). After 1 month of daily culturing on agar plates, no micro-organism growth was seen in both groups.

Conclusion: Topical anaesthetic eye drops can be used safely for 1 month in different patients after opening the bottle without any risk of causing infectious keratitis, conjunctivitis or endophthalmitis.

Keywords: Proparacaine, Culture medium, Endophthalmitis.
$\mathrm{T}$ opical anaesthetic eye drops are used extensively in ophthalmology. These agents provide sufficient corneal and conjunctival anaesthesia for comfortable performance of different examination techniques like tonometry, biometry, gonioscopy, contact lens fundoscopy and Nd-YAG laser posterior capsulotomy. Apart from examination techniques, these agents are used for certain therapeutic purposes as well; like removal of superficial corneal and conjunctival foreign bodies, removal of stitches and during surgeries of smaller duration like cataract extraction by phacoemulsification ${ }^{1}$.

Topical anaesthetics used in ophthalmology are tertiary amines linked by either ester or amide bonds to an aromatic residue. These act by blocking nerve impulse conduction by decreasing sodium permeability across the cell membranes ${ }^{1}$. These agents are weak bases, are more soluble in protonated form and their hydrolysis is slow in acidic environment so these are bound with hydrochloride ${ }^{1}$. Proparacaine is 
the preferred topical anaesthetic used for ophthalmological purposes as it causes lesser pain on instillation and longer anesthesia ${ }^{2}$.

Topical medications are easy to get contaminated and the rate of contamination is directly related to multiple use and also to duration for which the drops are being used ${ }^{3,4}$.

These drops are used extensively in Ophthalmology outdoor departments and also during various operative procedures which are known to have potentially vision threatening infectious complications like endophthalmitis. The rationale of the study was to check if these drops are a potential cause of endophthalmitis or other infections of lesser severity like conjunctivitis and keratitis. The purpose of the study was to determine the risk of ocular infections due to topical anaesthetic eye drops in patients one month after opening the bottle.

\section{MATERIALS AND METHODS}

This quasi experimental study was conducted after approval from the ethical and research committee of DHQ Teaching Hospital, Gujranwala/Gujranwala Medical College. The bottle contents of a topical formulation containing Proparacaine hydrochloride $(0.5 \%)$ as the anaesthetic agent and benzalkonium chloride $(0.01 \%)$ as a preservative used in Ophthalmology department of DHQ University teaching hospital, Gujranwala was tested on daily basis to check if any growth occurs or not. One bottle of Alcaine ${ }^{\circledR}$ eye drops was labelled as "study case" for this purpose and drops were instilled daily in eyes of different patients, one from the outdoor patient department and the other in the operation theater. Thus, 60 patients were included in our study and they were divided in two groups comprising of 30 patients in each. Group A included those who presented in the outdoor department and group B comprised of those presenting for various operative procedures. All patients were adults (18-60 years). Gender of the patients was not taken as a measure. Patients were selected through random sampling technique. Those who already had keratitis, conjunctivitis, corneal opacities, thin corneas and having dry eyes, which were prone to get infection were excluded from this study. Great care was taken while instilling eye drops, not to touch tip of the bottle to the ocular surface, adnexa or any other thing to avoid any possible contamination, which could give false positive culture results. After instilling drops, the bottle was capped carefully and tightly before taking any samples for culture growth and stored in a refrigerator afterwards.

In the laboratory, sample was applied on blood agar, MacConkey agar and Sabouraud agar following the conventional techniques. Blood agar and MacConkey agar plates were incubated at $37^{\circ} \mathrm{C}$ for 24 hours whereas Sabouraud agar plate was incubated at $37^{\circ} \mathrm{C}$ for $24-48$ hours to provide adequate time and environment for bacterial and fungal growth respectively. Samples were cultured daily for 30 days consecutively. All the patients were followed up on day 1 , day 7 and day 30 to see if any microbial infection had occurred or not.

\section{RESULTS}

More than half $(58 \%)$ of the patients in group A presented with complaint of superficial corneal/ conjunctival foreign body (Figure 1). There were 14 of $30(47 \%)$ patients in group B who were candidates of cataract extraction by phacoemulsification (Figure 2). There were 37 of $60(61.6 \%)$ patients who complained of transient mild irritation/stinging after instilling the anaesthetic eye drops while 17 of 60 (28.3\%) were noted to have increased blinking. Others complained of redness and increased lacrimation (Table 1). All these complaints were short-lived and subsided within an hour.

All patients were followed up on already defined times and sampling of eye drops was done daily for 30 days. No signs of bacterial or fungal infection was noted in any of these patients and no microbial growth was seen on the culture media we used in our study (Table 2).

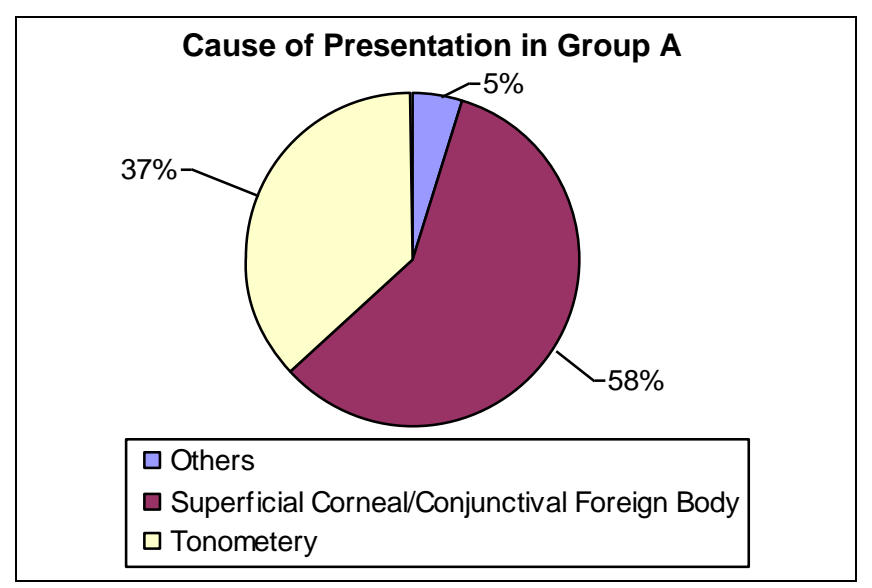

Fig. 1: Cause of presentation in group A (outdoor patients). 


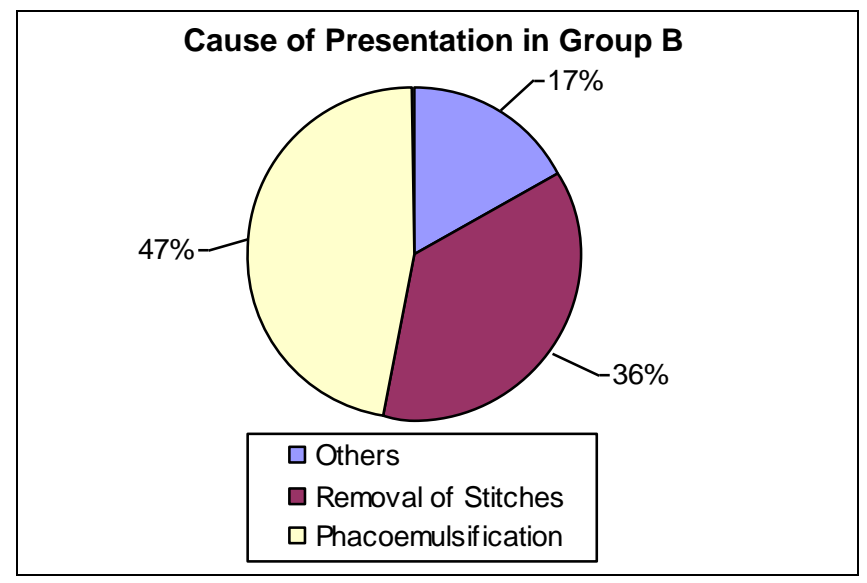

Fig. 2: Cause of presentation in group B (Operation Theater patients).

Table 1: Complaints while instilling the topical anesthetic drops.

\begin{tabular}{|lcc|}
\hline $\begin{array}{l}\text { Complaints While Instilling } \\
\text { the Drops }\end{array}$ & $\begin{array}{c}\text { No. of } \\
\text { Patients }\end{array}$ & Percentage \\
\hline Irritation/Stinging & 37 & $61.6 \%$ \\
Redness & 5 & $8.3 \%$ \\
Increased lacrimation & 11 & $18.3 \%$ \\
Increased blinking & 17 & $28.3 \%$ \\
\hline
\end{tabular}

Table 2: Results of growth on different culture media on consecutive 30 days.

\begin{tabular}{|lc|}
\hline Culture Medium & Growth on Day 1 - 30 \\
\hline MacConkey agar & No \\
Blood agar & No \\
Sabouraud agar & No \\
\hline
\end{tabular}

\section{DISCUSSION}

Topical anesthetics eye drops are widely used in Ophthalmology for various outdoor and indoor procedures of short duration; removal of superficial corneal and conjunctival foreign bodies, tonometry, phacoemulsification etc. These agents provide sufficient anaesthesia to carry out these procedures under comfortable circumstances both for the patient and the doctor ${ }^{1}$. Topical anaesthesia has largely replaced other techniques of local anesthesia like retrobulbar/peribulbar anaesthesia being employed during cataract extraction via phacoemulsification ${ }^{5-7}$. A study conducted by Rong $\mathrm{Han} \mathrm{Wu}$ and co-authors has found topical anaesthesia as a safer alternative to peribulbar/retrobulbar anaesthesia for patients undergoing pars plana vitrectomy ${ }^{8}$.
It is a common practice to use a single bottle of topical formulations in different patients with different complaints. Asegedech Tsegaw and colleagues found out that multi-dose eye drops are easily contaminated due to frequent and long-term usage and may result in inadvertent damage to ocular surfaces by causing unwanted infections ${ }^{9}$. Nentwich $\mathrm{MM}$ and co-authors conducted a similar study in Kenya on 101 bottles of multi-use and single-use topical ophthalmic solutions with similar conclusions ${ }^{10}$. The rate of contamination is variable in literature and it ranges from as low as $0.07 \% 11$ to as high as $35.8 \%^{12}$ and is directly related to the increased duration of use ${ }^{4}$. A study conducted by Mohammad Reza Fazeli et al. stated that topical anesthetic eye drops were the most commonly contaminated drops among all other multi-use topical formulations ${ }^{13}$. Contamination of the topical formulations is the source of different ocular infections. The most severe of these is Endophthalmitis, which is infective inflammation of the intraocular structures and is potentially sight-threatening ${ }^{14}$.

Topical anaesthetic eye drops are easily available as an over-the-counter drug and people are likely to get addicted to these medications because of the temporary relief these agents provide in case of ocular surface irritation. Some of the medical professionals (the primary care practitioners) also recommend these as routine medication for this purpose without knowing the adverse effects these can cause on ocular surface integrity ${ }^{15}$. Cases have been reported where the use of topical anaesthetics over long time periods have been linked to severe damage to eyes in the form toxic keratopathy and infections ${ }^{16-18}$.

In a previous study, it was found that eye drops can be used safely in the hospital settings for up to 2 weeks without being a risk factor for ocular infections ${ }^{4}$. In our study, we extended the time period upto 30 days. The reported source of infection in most of the literature review is contaminated tip 3,9-12 and contamination of the bottle contents ${ }^{12}$. In our study, we cultured only the bottle contents.

Garcia-Arumi and co-authors have conducted a similar retrospective study on patients undergoing cataract surgery and have found topical anesthesia to be related to postoperative endophthalmitis with an odds ratio [OR] of 11.8 and $95 \%$ confidence interval $[\mathrm{CI}]^{19}$. Hou-Chuan Lai and co-authors have also conducted a retrospective study comparing different types of anaesthesia for intraocular surgeries and found out that the rate of postoperative 
endophthalmitis after phacoemulsification is significantly higher for topical anaesthesia as compared to general anaesthesia $(0.083: 0)^{20}$.

The results of our study are in contrast to all the similar studies conducted in the past as no growth was observed in the bottle contents and no signs of infection was noted in the eyes of patients in whom the drops were instilled. The limitation of the study is small sample size and a similar study is needed to be conducted on a larger scale considering other risk factors like time of presentation to the doctor, duration of surgery and the frequency and technique of instillation of eye drops to carry out different procedures with full convenience. We also recommend to carry out this research on a large scale so as to get more references in this regard.

\section{CONCLUSION}

On the basis of results of our study, we conclude that Alcaine ${ }^{\circledR}$ eye drops remain sterile for 1 month and can be used safely in different patients for this duration without any risk of causing bacterial or fungal infections, if care is taken while instilling the drops as previous studies have documented tip contamination as a source of ocular infections. This is also beneficial from economical point of view.

\section{Conflict of Interest}

The authors have no conflicts of interest to disclose regarding this study.

\section{REFERENCES}

1. Kumar M, Chawla R, Goyal M. Topical anesthesia. J Anaesthesiol Clin Pharmacol. 2015; 31 (4): 450-456. Doi:10.4103/0970-9185.169049.

2. Bartfield JM, Holmes TJ, Raccio-Robak N. A comparison of proparacaine and tetracaine eye anesthetics. Acad Emerg Med. 1994; 1 (4): 364-367.

3. Tamrat L, Gelaw Y, Beyene G, Gize A. Microbial Contamination and Antimicrobial Resistance in Use of Ophthalmic Solutions at the Department of Ophthalmology, Jimma University Specialized Hospital, Southwest Ethiopia. Can J Infect Dis Med Microbiol. 2019, Article ID 5372530, 8 pages,

4. Livingstone DJ, Hanlon GW, Dyke S. Evaluation of an extended period of use for preserved eye drops in hospital practice. Br J Ophthalmol. 1998; 82 (5): 473-475. Doi:10.1136/bjo.82.5.473.

5. Guay J, Sales K. Sub-Tenon's anaesthesia versus topical anaesthesia for cataract surgery. Cochrane Database of
Systematic Reviews, 2015, Issue 8. Art. No.: CD006291. DOI:10.1002/14651858.CD006291.pub3.

6. Jacobi PC, Dietlein TS, Jacobi FK. A Comparative Study of Topical vs. Retrobulbar Anesthesia in Complicated Cataract Surgery. Arch Ophthalmol. 2000; 118 (8): 1037-1043. Doi:10.1001/archopht.118.8.1037.

7. Malik A. Efficacy and Performance of Various Local Anesthesia Modalities for Cataract Surgery. J Clinic Experiment Ophthalmol. 2013; S1: 007.

Doi:10.4172/2155-9570.S1-007

8. Wu RH, Zhang R, Lin Z, Liang QH, Moonasar N. A comparison between topical and retrobulbar anesthesia in 27-gauge vitrectomy for vitreous floaters: a randomized controlled trial. BMC Ophthalmol. 2018; 18 (1): 164

9. Tsegaw A, Abula T, Assefa Y. Bacterial Contamination of Multi-dose Eye Drops at Ophthalmology Department, University of Gondar, Northwest Ethiopia. Middle East Afr J Ophthalmol. 2017; 24 (2): 81-86. Doi:10.4103/meajo.MEAJO_308_16.

10. Nentwich MM, Kollmann KH, Meshack J, Ilako DR, Schaller UC. Microbial contamination of multi-use ophthalmic solutions in Kenya. Br J Ophthalmol. 2007; 91 (10): 1265-1268. Doi:10.1136/bjo.2007.116897.

11. Wessels IF, Bekendam P, Calvin WS, Zimmerman J. Open drops in ophthalmology offices: expiration and contamination. Ophthalmic Surg Lasers 1999; 30: 540546.

12. Brudieu E, Duc DL, Masella JJ, Croize J, Valence B, Meylan I, et al. Bacterial contamination of multi-dose ocular solutions. A prospective study at the Grenoble Teaching Hospital. Pathol Biol (Paris), 1999; 47: 10651070.

13. Fazeli MR, Nejad HB, Mehrgan H, Elahian L, "Microbial contamination of preserved ophthalmic drops in outpatient departments: possibility of an extended period of use," DARU J Pharm. Sci.2004; 12 (4): 151-155.

14. Durand ML. Bacterial and Fungal Endophthalmitis. Clin Microbiol Rev. 2017; 30 (3): 597-613.

Doi:10.1128/CMR.00113-16

15. Erdem E, Undar IH, Esen E, Yar K, Yagmur M, Ersoz R. Topical anesthetic eye drops abuse: are we aware of the danger? Cutan Ocu Toxicol. 2013; 32 (3): 189-193.

16. Aksoy A, Başkan AM, Aslan L, Aslankurt M. Topical proparacaine abuse resulting in evisceration. BMJ Case Rep. 2013; 2013: bcr2013009539. Published 2013 Apr 22. Doi:10.1136/bcr-2013-009539

17. Tok OY, Tok L, Atay IM, Argun TC, Demirci N, Gunes A. Toxic keratopathy associated with abuse of topical anesthetics and amniotic membrane transplantation for treatment. Int J Ophthalmol. 2015; 8 (5): 938-944. Doi:10.3980/j.issn.2222-3959.2015.05.15

18. Kintner JC, Grossniklaus HE, Lass JH, Jacobs G. Infectious crystalline keratopathy associated with topical anesthetic abuse. Cornea. 1990; 9 (1): 77-80.

19. Garcia-Arumi J, Fonollosa A, Sararols L, Fina F, 
Martínez-Castillo V, Boixadera A et al. Topical anesthesia: possible risk factor for endophthalmitis after cataract extraction. J Cataract Refract Surg. 2007; 33(6): 989-92.

\section{Author's Affiliation}

Dr. Mehr-un-Nisa

Postgraduate Resident

MBBS

Ophthalmology Department

DHQ Teaching Hospital, Gujranwala.

Dr. Irfan Qayyum

Associate Professor

MBBS, FCPS, FVR

Ophthalmology department

DHQ Teaching Hospital, Gujranwala
20. Lai HC, Tseng WC, Pao SI, Wong CS, Huang RC, Chan WH et al. Relationship between anesthesia and postoperative endophthalmitis: a retrospective study. Medicine, 2017; 96: e6455.

Dr. Muhammad Wasif Irshad

House Officer

MBBS, Ophthalmology Department

DHQ Teaching Hospital, Gujranwala.

\section{Author's Contribution}

Dr. Mehr-un-Nisa

Study design and manuscript writing.

Dr. Irfan Qayyum

Manuscript review and critical analysis.

Dr. Muhammad Wasif Irshad

Data collection and manuscript writing. 